\title{
Repurposing an ancient protein core structure: structural studies on FmtA, a novel esterase of Staphylococcus aureus
}

\author{
V. Dalal ${ }^{1}$, P. Kumar ${ }^{1}$, G. Rakhaminov², A. Qamar' ${ }^{2}$ X. Fan², H. Hunter², S. Tomar ${ }^{1}$, D. G. Kotra ${ }^{2}$, and P. Kumar ${ }^{1 *}$ \\ ${ }^{I}$ Department of Biotechnology, IIT Roorkee, Uttrakhand-247667, India affiliation, , \\ ${ }^{2}$ Department of Biology, York University, 4700 Keele Street, Toronto, Canada \\ vdalal@bt.iitr.ac.in
}

FmtA is a penicillin-recognizing protein (PRP) with novel hydrolytic activity toward the ester bond between D-Ala and the backbone of teichoic acids (TA), the polyol-phosphate polymers found in the $S$. aureus cell envelope. Two of the PRPs conserved motifs, namely SXXK and $\mathrm{Y}(\mathrm{S}) \mathrm{XN}$, are involved in this hydrolysis, but its catalytic mechanism remains elusive. Here we determined the crystal structure of FmtA. FmtA shares the core structure of PRPs: an all $\alpha$-helical domain and $\alpha / \beta$ domain sandwiched together. However, it does not have the typical PRPs active-site cleft. Its active site is shallow, solvent-exposed and wide. Furthermore, the SXXK and Y(S)XN motifs of FmtA offer all that is necessary for catalysis: the active-site nucleophile (serine) and the general base (lysine) required for acylation and deacylation steps and an anchor (tyrosine) to hold the active-site serine, and possibly the substrate, in an optimum conformation for catalysis [1]. Our study establishes that the FmtA esterase activity represents an expansion of the catalytic activity repertoire of the PRPs core structure, which typically displays peptidase activity. The structure of FmtA provides insights to the design of inhibitor molecules with the potential to serve as leads in the development of novel antibacterial chemotherapeutic agents.

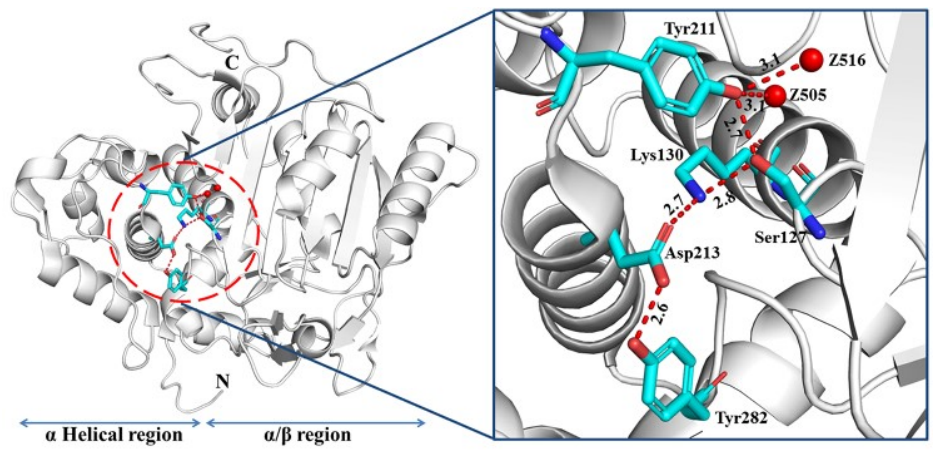

Figure 1. Cartoon representation of the FmtA structure with the active-site residues shown in sticks.

[1] Dalal, V., Kumar, P., Rakhaminov, G., Qamar, A., Fan, X., Hunter, H., Tomar, S., Golemi-Kotra, D. and Kumar, P., (2019). Journal of molecular biology, 431(17), pp.3107-3123.

Keywords: teichoic acids; FmtA; Staphylococcus aureus; penicillin-recognizing proteins. 